\title{
ANÁLISE DA EPIDEMIĀ PELA COVID-19 A PARTIR DO MODELO DOS MÚLTIPLOS FLUXOS DE KINGDON
}

Lucas Dias Soares Machado ${ }^{1}$ Samyra Paula Lustoza Xavier ${ }^{2}$ Francisco Ayslan Ferreira Torres ${ }^{2}$ Paloma Loiola Leite ${ }^{2}$ Tiago Ribeiro dos Santos ${ }^{2}$ Larissa Uchôa Melo² Sabrina Freitas Nunes ${ }^{2}$ Maria Rocineide Ferreira da Silva https://orcid.org/0000-0003-4450-3796 https://orcid.org/0000-0002-5295-7627 https://orcid.org/0000-0002-1703-0025 https://orcid.org/0000-0002-2380-8462 https://orcid.org/0000-0002-8614-0818 https://orcid.org/0000-0001-9026-3303 https://orcid.org/0000-0003-1442-0092 https://orcid.org/0000-0002-6086-6901

Objetivo: analisar o fluxo de problemas, soluções e políticas no enfrentamento a epidemia pela COVID-19 no Ceará, com base no Modelo de Múltiplos Fluxos de Kingdon. Método: estudo misto com dados provenientes dos indicadores, de 55 documentos oficiais de saúde e de 108 matérias jornalísticas veiculadas na mídia digital, de fevereiro a abril de 2020. Resultados: a análise do fluxo problemas aponta uma expansiva interiorização dos casos confirmados da doença, com perspectiva de elevação do número de internações e óbitos; o fluxo de soluções sinaliza que a adoção de medidas preventivas e protetivas contra a doença foram ampliadas concomitantemente à progressão da doença no estado, e por fim, o fluxo de políticas evidenciou que o Ceará tem direcionado suas ações de forma positiva, alinhando parcerias e alavancado estratégias para minimização dos problemas provocados pela COVID-19. Conclusão: os fluxos que perfazem as tomadas de decisão no enfrentamento a pandemia da COVID-19 no Ceará mostram-se complexos e amplos, estando em sua maioria alinhados com posicionamentos de países que obtiveram êxito nesse enfrentamento. Urge a necessidade de estabelecer parcerias efetivas entre atores, serviços e setores instigados.

Descritores: Infecções por Coronavírus; Pandemias; Estudos de Avaliação.

\section{ANALYSIS OF PANDEMIC BY COVID-19 FROM THE MODEL OF THE MULTIPLE FLOWS OF KINGDON}

Objective: to analyze the flow of problems, solutions and policies in facing the epidemic by COVID-19 in Ceará, based on Kingdon's Multiple Streams Model. Method: mixed study with data from the indicators, 55 official health documents and 108 journalistic articles published in digital media, from February to April 2020. Results: the analysis of the problem flow points to an expansive interiorization of confirmed cases of the disease, with the prospect of increasing the number of hospitalizations and deaths; the flow of solutions signals that the adoption of preventive and protective measures against the disease was expanded concomitantly with the progression of the disease in the state, and finally, the flow of policies showed that Ceará has directed its actions in a positive way, aligning partnerships and leveraging strategies to minimize the problems caused by COVID-19. Conclusion: the flows that make up the decision-making in coping with the COVID-19 pandemic in Ceará are complex and broad, being mostly aligned with the positions of countries that have been successful in this confrontation. There is an urgent need to establish effective partnerships between actors, services and instigated sectors.

Descriptors: Coronavirus infections; Pandemics; Evaluation Studies.

\section{ANÁLISIS DE LA EPIDEMIA POR COVID-19 UN SOCIO EN EL MODELO DE FLUJOS MÚLTIPLES DE KINGDON}

Objetivo: analizar el flujo de problemas, soluciones y políticas para enfrentar la epidemia por COVID-19 en Ceará, basado en el Modelo de Corrientes Múltiples de Kingdon. Método: estudio mixto con datos de los indicadores, 55 documentos oficiales de salud y 108 artículos periodísticos publicados en medios digitales, de febrero a abril de 2020. Resultados: el análisis del flujo del problema apunta a una internalización expansiva de casos confirmados de la enfermedad, con la perspectiva de aumentar el número de hospitalizaciones y muertes; El flujo de soluciones indica que la adopción de medidas preventivas y protectoras contra la enfermedad se expandió concomitantemente con la progresión de la enfermedad en el estado, y finalmente, el flujo de políticas mostró que Ceará ha dirigido sus acciones de manera positiva, alineando alianzas y apalancamiento estrategias para minimizar los problemas causados por COVID-19. Conclusión: los flujos que conforman la toma de decisiones para hacer frente a la pandemia COVID-19 en Ceará son complejos y amplios, en su mayoría alineados con las posiciones de los países que han tenido éxito en esta confrontación. Existe una necesidad urgente de establecer asociaciones efectivas entre actores, servicios y sectores instigados.

Descriptores: Infecciones por coronavirus; Pandemias; Estudios de evaluación.

'Universidade Estadual do Ceará, Ceará.

2Universidade Regional do Cariri, Ceará.

Autor correspondente: Lucas Dias Soares Machado E-mail: lucasdsmachado@hotmail.com

Recebido: 25/4/2020 Aceito: 28/5/2020 


\section{INTRODUÇÃO}

O final do ano de 2019 anunciou um novo desafio às sociedades e sistemas de saúde em todo o mundo: o surgimento de casos de infecção respiratória por um patógeno desconhecido em habitantes e visitantes de Wuhan, província de Hubei, na China, em dezembro. O novo patógeno logo foi reconhecido pelos cientistas chineses como sendo um novo tipo do coronavírus, - SARS-COV-2, e a doença ocasionada por ele foi denominada pela Organização Mundial de Saúde (OMS) como COVID-19 ${ }^{1}$.

Em 30 de janeiro de 2020, a importação dos casos do novo coronavirus para diversos continentes e países, dentre eles Estados Unidos, Canadá e Austrália, levou a OMS a classificá-la como uma Emergência de Saúde Pública de Âmbito Internacional ${ }^{2}$. Assim, a COVID-19 entra para lista de pandemias do século XXI, junto às epidemias de ebola, gripe, cólera, tuberculose e tifo. Pandemias compreendem doenças infecciosas de ampla disseminação que acontecem praticamente ao mesmo tempo em grandes regiões e diversos países, especialmente os latinomericanos, com impactos em diversos setores, especialmente na saúde e economia ${ }^{3-4}$.

A periculosidade da COVID-19 associa-se a sua alta transmissibilidade e fácil disseminação, ocasionando Síndrome Respiratória Aguda Grave com potencial de mortalidade por danos alveolares maciços e insuficiência pulmonar progressiva ${ }^{5}$. Assim, o enfrentamento desta epidemia tem produzido fortes desafios aos líderes de estado, gestores, profissionais de saúde e população em geral, posto ao desconhecimento real da problemática.

Sob este prisma, e considerando o aspecto inicial e recente da epidemia no Brasil, com primeiro caso confirmado em 26 de fevereiro de 2020, importado da Itália ${ }^{6}$, faz-se necessário a adoção de medidas preventivas e protetivas em todo o país, a fim de limitar a propagação da doença, achatar a curva epidemiológica de número de casos, embasar a organização do Sistema Único de Saúde (SUS) e orientar profissionais de saúde e a população.

Frente a estes desafios, o estado do Ceará tem demonstrado iniciativas significativas para o enfrentamento da situação sanitária em seu território, uma vez que este já encontra-se em situação de emergência, junto a São Paulo, Rio de Janeiro, Amazonas, Amapá e Distrito Federal, estando em terceiro lugar em número de casos confirmados da doença no país ${ }^{7}$.

Sabe-se que há influência de forças diversas no desenvolvimento de políticas públicas de saúde e tomada de decisões no âmbito do SUS, permeadas por ambiguidade, incertezas e conflitos entre valores e interesses de grupos de escolha, já comuns e agora acentuados frente a progressão da pandemia. Questiona-se, então: como o Ceará tem lidado com a crise sanitária pela COVID-19 e quais as forças mobilizadas neste processo?

Conhecer o fluxo de forças envolvidas na estruturação de políticas de saúde e tomadas de decisão frente a pandemia da
COVID-19 possibilita aproximar-se dos problemas existentes, das soluções já reconhecidas e adotadas e do processo político que permeia o enfrentamento da crise, viabilizando a análise da situação apresentada em comparação com outras realidades e, deste modo, contribuir na definição e divulgação de novas estratégias.

Assim, objetivou-se analisar o fluxo de problemas, soluções e políticas no enfrentamento à epidemia pela COVID-19 no Ceará, com base no Modelo de Múltiplos Fluxos de Kingdon.

\section{MÉTODO}

\section{Tipo de Estudo}

Estudo descritivo, com abordagem mista, compreendida como a vinculação entre a pesquisa quantitativa, onde buscam-se os indicadores e tendências de modo a se alcançar uma leitura do fenômeno, e pesquisa qualitativa, que possibilita o aprofundamento dos fenômenos, fatos e processos singulares. Nesta abordagem, optou-se pela distribuição temporal explanatória sequencial OUAN $\rightarrow$ qual $^{8}$, onde os dados quantitativos são analisados em um momento inicial da pesquisa, seguido pela análise dos dados qualitativos.

\section{Referencial teórico}

Utilizou-se como referencial teórico o Modelo dos Múltiplos Fluxos de Kingdon. Este modelo discute a tomada de decisão em políticas públicas a partir da contemplação de três fluxos de forças: o fluxo dos problemas, que envolve as questões reconhecidas e os motivos que as levam a compor a agenda do governo; 0 fluxo das soluções, propostas ou alternativas, que inclui as ideias para ganhar aceitação na rede de políticas e que propõem-se a resolução da problemática; e os fluxos políticos, delineados pelo clima nacional, forças políticas organizadas e mudanças no governo 9,10 .

Em suma, este modelo compreende a análise de um problema reconhecido pela sociedade em geral, das alternativas para conceituá-lo e enfrentá-lo e, por fim, do contexto político, administrativo e legislativo favorável a ação ${ }^{10}$.

\section{Local e periodo}

Adotou-se como lócus de análise o estado do Ceará, situado na região Nordeste do Brasil. O estudo foi desenvolvido durante o mês de abril de 2020 .

\section{Coleta de Dados}

Para coleta dos dados, adotou-se a estratégia de triangulação concomitante. Os dados referentes aos indicadores de saúde, aqui representados como fluxo de problemas, foram fornecidos pelas plataformas estratégicas Coronavirus Brasil (https:// covid.saude.gov.br/) e IntegraSUS (https://integrasus.saude.ce. gov.br/), publicados no dia 23 de abril de 2020, responsáveis por condensar diariamente os dados referentes a epidemia no Brasil e Ceará, respectivamente. 
Os materiais referentes ao fluxo de soluções foram selecionados a partir de documentos estaduais com base epidemiológica e de educação em saúde, compreendendo a seleção, extração e organização dos dados do Plano Estadual de Contingência para Respostas às Emergências em Saúde Pública - Novo Coronavírus (2019-nCoV), dos boletins epidemiológicos ( $\mathrm{n}=32$ ), iniciados em 27 de fevereiro de 2020, e do material informativo na forma de cartilhas e cartazes ( $\mathrm{n}=33$ ) produzidos e divulgados pela Secretaria Estadual de Saúde do Ceará a partir de então.

Os fluxos políticos foram resgatados por meio de textos jornalísticos dos dois maiores jornais impressos e online de consumo pela população cearense, a saber: o jornal O Povo e o Diário do Nordeste. Para tanto, foram selecionadas matérias disponíveis ( $\mathrm{n}=108$ ) nos sítios eletrônicos destes jornais por meio de busca simples, que versassem sobre a COVID-19. As matérias selecionadas subsidiaram ainda a complementação dos fluxos das soluções, visto que medidas intersetoriais estão sendo adotadas no processo de enfrentamento a pandemia e não estão registradas nos documentos da Secretaria Estadual de Saúde, sendo relevantes para compreensão ampla da situação.

\section{Procedimentos de análise e tratamento dos dados}

Para o tratamento dos dados representantes dos fluxos dos problemas utilizou-se o Office Excel® 2016 na organização em planilha eletrônica e o software R Studio versão 3.2.1 para análise descritiva, expressa pelos indicadores de magnitude e de ocorrência de eventos, calculando-se as médias, medianas e proporções. Utilizou-se ainda o Open Street Maps para representação cartográfica dos casos no Ceará, por meio de mapa analítico de calor estático

A organização dos dados qualitativos, oriundos dos documentos estaduais oficias e textos jornalísticos, se deu por meio da análise de conteúdo, do tipo temática, fundamentada na descoberta de núcleos de sentido que compõem uma comunicação; e codificação hierárquica desses temas, para proporcionar síntese taquigráfica ${ }^{11}$, considerando-se a essência da ideia para representar cada um dos três fluxos.

\section{Aspectos éticos}

O estudo fez uso de dados de acesso público e gratuito, disponíveis em meio eletrônico, isentando-o da necessidade de apreciação por Comitê de Ética em Pesquisa. No entanto, para garantir a neutralidade na análise das informações, adotou-se códigos como $\mathrm{Jl}$ e $\mathrm{J} 2$ para os textos jornalísticos utilizados e Doc para os documentos oficiais da Secretaria Estadual de Saúde do Ceará

\section{RESULTADOS}

Esta seção traz uma análise da pandemia pela COVID-19 no Ceará, considerando-a o problema mor que desecandeou uma série de ações e mobilização de forças distintas, por vezes opostas, em sua minimização e/ou resolução no território cearense.

A sintese dos fluxos dos problemas, das soluções e políticos envolvidos no enfrentamento a pandemia pela COVID-19 no Ceará está ilustrada por meio da Figura 1, segundo o modelo de múltiplos fluxos de Kingdon.

Figura 1 - Síntese dos fluxos dos problemas, soluções e políticos mobilizados no enfrentamento a pandemia no Ceará, 2020.
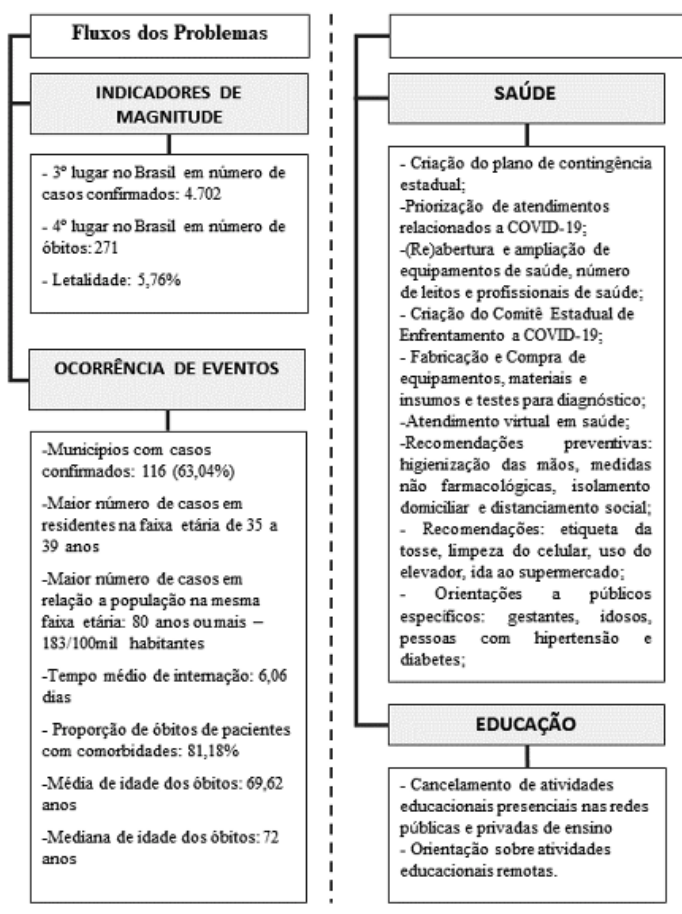

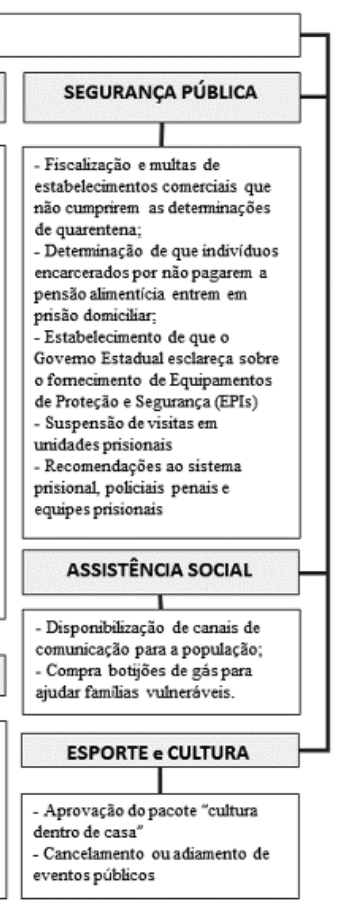

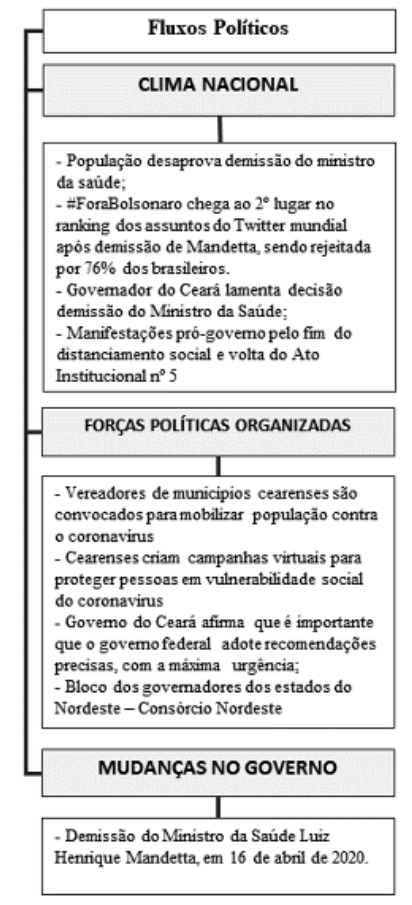


Em um panorama nacional, até 23 de abril de 2020, o Brasil registrou a confirmação de 49.492 casos de COVID-19. Destes, 3.313 evoluíram com prognóstico de óbito, representando uma taxa de letalidade de 6,7\% em território nacional.

Na região Nordeste, segunda em número de casos confirmados no país, o Ceará tem contribuído significativamente no número de casos, contemplando 4.702 casos confirmados, acrescidos de 14.750 casos suspeitos em investigação. Destes, 271 pessoas acometidas com a COVID-19 evoluíram à óbito, representando uma taxa de letalidade de 5,76\% e uma média de 6,95 óbitos por dia.

A análise do fluxo problemas evidencia que o Ceará, considerando a distribuição espacial dos casos, representado na Figura 2, vivencia uma significativa expansão da epidemia, com forte concentração inicial na capital Fortaleza e municípios circunvizinhos, e atual interiorização da doença, com notificação de casos em municípios polos como Sobral, Quixadá, Iguatu, Limoeiro do Norte e Juazeiro do Norte, totalizando 116 casos distribuídos em 184 municipios cearenses $(63,04 \%)$

Figura 2 - Mapa de Calor Estático dos casos de COVID-19 no estado do Ceará entre 20 de março e 20 de abril de 2020.

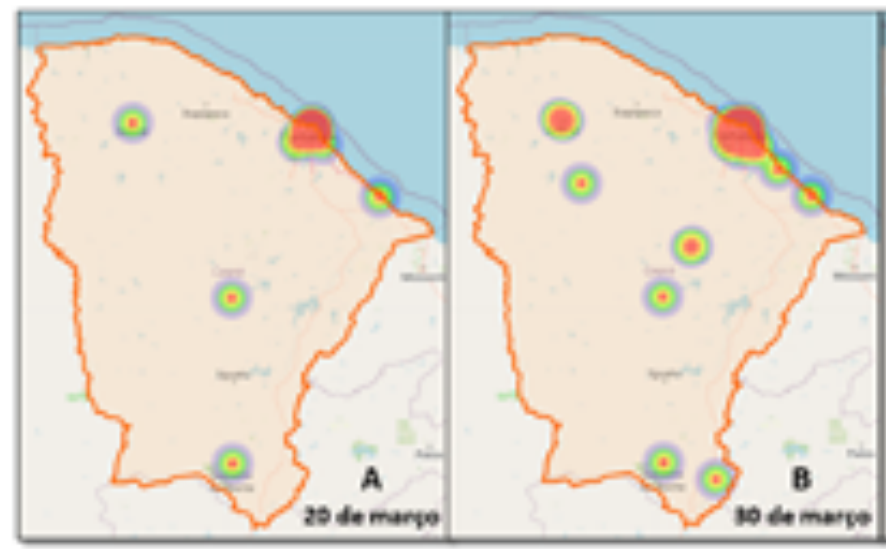

Fonte: IntegraSUS.

A definição dos aspectos condizentes ao fluxo das soluções para enfretamento a pandemia no Ceará, tem demonstrado posicionamento de antecipação à chegada do vírus, quando, antes da confirmação do primeiro caso no estado, o Ceará já tinha o seu Plano de Contingência para COVID-19 e já iniciava a adoção de medidas preventivas, monitoramento dos casos suspeitos e lançamento de estratégias para combater o coronavirus.

Após confirmação do primeiro caso no estado, em 15 de março, houve ampliação das recomendações de cuidados e adoção de medidas de proteção populacional, visando a redução da propagação do vírus na população e o achatamento da curva epidemiológica para níveis de resposta efetiva dos serviços de saúde existentes, a partir de uma projeção epidemiológica feita por especialistas, os quais sinalizaram a ascensão no número de casos nos dias/meses seguintes.

A adoção de medidas preventivas e protetivas evoluiu conforme propagação da doença, apresentando flutuações, onde as medidas de cuidados primários, recomendadas, inicialmente, de maneira genérica e destinada a grupos de risco, deram espaço as medidas de isolamento horizontal, fortemente marcadas pela recessão nas atividades do comércio, educação, cancelamento de eventos culturais, esportivos, dentre outros (Figura 1).

Entretanto, em Fortaleza manifestações foram articula- das contra o isolamento decretado e renovado pelo governador, conforme publicado:

"Carreata contra isolamento social em Fortaleza [...] Manifestantes expressaram insatisfação com o decreto estadual que impõe série de restrições ao funcionamento do comércio e ao convívio social." (JI)

Além da resistência em seguir as mediadas recomendadas, o Ceará apresenta ainda o desafio na identificação dos casos confirmados para início da quarentena destes, pois o limitado número de testes rápidos disponíveis soma-se as dificuldades da rede pública de laboratórios em sanar o acumulado de exames diagnósticos existentes.

“O exame NÃO está indicado para pessoas sem sintomas da doença e para casos de resfriado ou Sindrome Gripal sem fatores de risco elou comorbidades. Os casos priorizados serão os sintomáticos classificados com a forma grave da doença Sindrome Respiratória Aguda Grave e óbitos." (Doc)

Cabe pontuar a resposta célere do estado do Ceará em mobilizar-se para melhor equipar seus serviços de saúde, informar a população através de material educativo e 
transparência de indicadores, implantar e garantir estado de quarentena e organização de medidas de auxílio a famílias em maior vulnerabilidade econômica e social. Em um sentido amplo, as soluções propostas têm demonstrado a transversalidade da condição de saúde com outros aspectos do viver, tais como trabalho, política e economia (Figura 1), enfatizando as forças de tensão existentes entre esses campos.

Dentre essas forças, reconhece-se os aspectos de fluxo político que impactam sobre as decisões para o desenvolvimento da ação.

Os conflitos entre o Presidente e o Ministro da Saúde à época, que culminou na sua demissão, provocaram o descontentamento da população brasileira e de outros líderes politicos, uma vez que as decisões tomadas pelo primeiro, bem como seus discursos e ações contraditórias, trazem ainda mais insegurança e instabilidade nesse cenário de crise, conforme expresso nas reportagens:

"Com atuação forte em meio à crise do coronavírus, o senador cearense (..) considera que questões político-ideológicas têm prejudicado o Brasil na pandemia" (J2).

"A medida (Isolamento horizontal), defendida por Mandetta, visa prevenir o colapso do sistema público de saúde. Esse teria sido o grande motivo de atrito entre o ministro com o presidente, que é contrário às decisões" (J2).

É válido salientar que tal insatisfação extrapola os limites territoriais do Brasil, ganhando também espaço nas mídias internacionais.

“(...) o Brasil ganha espaço na midia internacional com sua crise de gestão governamental em

meio à escalada recorde das estatísticas de óbitos" (JI).

É neste clima de inquietação que as forças políticas locais se manifestam e se unem na tentativa de unificar e alinhar as propostas de combate ao coronavírus no Ceará. As inquietações abriram espaço para a união e o fortalecimento de parcerias, entre setores públicos, privados e demais esferas de liderança social, para além do setor saúde.

"Deputados e Governo Estadual no Ceará alinham medidas de enfrentamento ao coronavírus "(JI).
"Cearenses criam campanhas virtuais para proteger pessoas em vulnerabilidade social do coronavírus" (JI).

\section{DISCUSSÃO}

A análise do enfrentamento das forças mobilizadas no combate a pandemia pela COVID-19 no Ceará, demonstra a interrelação existente entre os fluxos dos problemas, das soluções e políticos, anunciados pela influência destas dimensões entre si, reverberando em alterações ora positivas, ora negativas, no panorama geral apresentado.

Os altos índices de COVID-19 no Ceará, especialmente na capital, estão relacionados ao fato de que a população residente nessa região tem mais chances de ter contato com alguém que esteve no exterior e que testou positivo para o coronavírus, considerando o papel turístico e econômico da cidade ${ }^{5}$.

A análise da pandemia enquanto fluxo de problemas fundamenta-se no reconhecimento de indicadores sociais e epidemiológicos expressivos no setor saúde para compreensão do destaque apresentando pela problemática de interesse, frente a tantas outras existentes, de modo que esta seja reconhecida socialmente e passe a compor a agenda do governo ${ }^{10}$.

O reconhecimento da COVID-19 enquanto emergência em saúde pública e sua presença no estado do Ceará, são forças que despertam a atenção dos atores envolvidos no processo saúde-doença-cuidado. Os crescentes índices de pessoas com confirmação de COVID-19 e do número de óbitos decorrentes dela, quando associados a medidas preventivas e protetivas em larga escala, como distanciamento social, isolamento horizontal e estado de quarentena, além da paralisação de atividades de diversos setores, representam o impacto social da pandemia.

A análise dos índices por meio do monitoramento de curvas epidemiológicas e indicadores de morbimortalidade possibilita o acompanhamento dos casos, antevendo o cenário epidemiológico e subsidiando a programação de medidas assistenciais necessárias a seu enfrentamento. A partir da compreensão que o aumento veloz da progressão da curva epidemiológica é proporcional ao aumento da necessidade de internações e, consequentemente, de leitos de terapia intensiva em intervalo curto de tempo, avalia-se a capacidade do sistema de saúde em responder a esta demanda ${ }^{12}$.

Esta problemática amplia a discussão para condições paralelas associadas ao possível agravamento da situação como superlotação, precarização dos serviços e insegurança dos profissionais de saúde devido à redução de Equipamentos de Proteção Individual (EPI) disponíveis, materializando proble- 
mas organizacionais de gestão e trabalho, que tem impacto direto na assistência à saúde em meio a pandemia ${ }^{13}$.

A discussão se aprofunda ainda mais ao se refletir sobre a pluralidade de realidades vividas pelo povo brasileiro, pois, considerando a dimensão territorial do país, as diferentes experiências que os estados vivem diante da mesma pandemia, os determinantes sociais e as iniquidades em saúde, enfatiza-se que, embora a suscetibilidade ao vírus seja transversal a toda a população, a vulnerabilidade ao adoecimento pela COVID-19 está diretamente ligada aqueles que estão expostos sistematicamente a pobreza, a doenças e à morte ${ }^{13-14}$.

As vulnerabilidades evidenciam-se nos modos de organização e vida dos brasileiros, exemplificadas pelo viver em favelas, assentamentos e ruas, com condições desfavoráveis ao distanciamento social preconizado, e diante de barreiras no acesso a condições essenciais à proteção contra o vírus, como a lavagem das mãos ${ }^{14}$.

Este reconhecimento é essencial para uma melhor percepção e interpretação da realidade, direcionando olhares para os diversos grupos, contextos e cenários de vulnerabilidade, voltando atenção às iniquidades em saúde e a mobilização de forças para solucionar, minimizar ou sanar tal problemática ${ }^{15-16}$.

No que tange ao isolamento social adotado, esta estratégia condiz com intervenções essenciais e urgentes na estrutura social para confronto a epidemia, com resultados positivos na redução da disseminação em países como a China ${ }^{1}$, sendo, atualmente, a principal estratégia para prevenção e contenção da COVID-19.

Entretanto, forças contrárias, defendidas inclusive em discursos sistemáticos e frequentes do Presidente da República, mobilizam-se para findar o isolamento físico social na defesa da segurança do desenvolvimento econômico do país, uma vez que seu modus operandi não apresenta sustentabilidade na forma neoliberal na qual se organiza, ruindo frente a suspensão, ainda que temporária, da circulação ${ }^{12,15}$.

Desvela-se uma crise neoliberal e democrática para além de uma desestabilização na saúde pública, ancorada na contradição de circulação mesmo com a expectativa do adoecimento e morte de parcela significativa da população decorrente disso ${ }^{15}$.

Complementar ao distanciamento físico social declamado, mostrou-se efetivo, na China, a proteção de profissionais de saúde com EPI em quantidade e qualidade adequados, a identificação dos casos sintomáticos e assintomáticos com a testagem e confirmação rápida, seguida da quarentena dos casos positivos e seus comunicantes ${ }^{17}$.

Ocorre então um subdimensionamento da curva epidêmica do Ceará, decorrente da minoração na sensibilidade diagnóstica pela testagem estar voltada apenas ao público prioritário, tais como pessoas hospitalizadas, profissionais de saúde e indivíduos com comorbidades, não contemplando sintomáticos leves e assintomáticos, que representam a maior parte dos infectados previstos. Sob este prisma, os esforços de contenção da epidemia estão fragilizados ${ }^{12}$.

Existem, então, duas forças interligadas que refletem sobre este panorama: os índices que não retratam a real face da epidemia pela não testagem eficiente da população, contribuindo para uma falsa sensação de segurança na população e potencial ampliação do contágio e, o planejamento em saúde, que carece de informações para melhor orientar profissionais e serviços na assistência a esses indivíduos e suas coletividades.

Neste panorama, manifestam-se ainda as forças políticas decisivas para a tomada de decisões oportunas e eficientes. A análise deste fluxo envolve o reconhecimento de elementos que se fundamentam na ambiguidade na tomada de decisão em função de três fatores, a rotatividade dos líderes, as preferências vulneráveis e disputas intra e intergovernamentais 9,10.

O clima nacional demarca-se nesse contexto pela reorganização das forças políticas conservadoras, fortalecimento da pauta antidemocrática e autoritária e forte mercantilização dos direitos sociais brasileiros, com ameaças a imprensa, desrespeito a minorias, incentivo a brutalidade policial e comprometimento da laicidade do Estado pelo protestantismo evangélico ${ }^{19-20}$.

A fluidez e a rotatividade dos atores e dos discursos nas arenas decisórias, sinalizam a instabilidade e o despreparo do governo em lidar com a crise que se instalou atualmente, tendo em vista que, apesar de o então Ministro da Saúde, estar seguindo as orientações da Organização Mundial da Saúde (OMS) no combate a pandemia no tocante aos isolamento social, não há o alinhamento com as ideias e propostas do principal líder da governança nacional.

É nítido que a formulação discursiva e prática de atores políticos, precedida pela figura do Presidente da República, desconsidera as contribuições científicas e o empenho de pesquisadores na produção de conhecimento sobre a pandemia e as possibilidades de solução, ilustrada pela desvalorização de narrativas ancoradas em evidências científicas e experiências de outros paises e disseminação de fake news que fragilizam o empenho da coletividade para enfretamento da situação instalada ${ }^{12}$.

Por fim, e não menos importante, o presidente subestima a epidemia de coronavirus, exemplificando o obscurantismo anticientífico de sua gestão e a presunção da extrema direita em relação a crise instaurada ${ }^{20}$.

Ainda que as forças políticas se encontrem em um campo 
de tensões, o Ceará tem direcionado suas ações de forma positiva, alavancado estratégias para minimização dos problemas, superação de omissões históricas quanto a iniquidades em saúde e organização dos serviços de saúde e ação para reestabelecimento e/ou efetivação da capacidade do sistema de saúde em vigiar, conter e mitigar esta epidemia. Enfrenta-se medidas políticas responsáveis por acentuar a desigualdade econômica, a precarização do trabalho, a limitação de políticas públicas e o enfraquecimento das ações e serviços de saúde, atinentes as vulnerabilidades ao coronavírus e suas consequências.

\section{Limitações do estudo}

Pode-se pontuar como limitações a baixa capacidade preditiva, frente a recente manifestação da problemática no país e estado, bem como a interpretação indutiva e dialógica da realidade transversal apresentada. Acrescenta-se que a adoção do modelo teórico dos múltiplos fluxos e a discussão com outros constructos teóricos foram empregadas para minimizar estas limitações.

\section{Contribuições para a Prática}

Analisar os fluxos de forças envolvidas na definição e apresentação de problemas, no empenho em mobilizar soluções para seu enfrentamento e dos embates existentes na dinâmica política, possibilita compreender o processo de formulação e efetivação de políticas públicas, entendendo como estas se estruturam e quais fatores são essenciais ao seu sucesso.

\section{CONSIDERAÇÕES FINAIS}

Os fluxos dos problemas, das soluções e políticos que perfazem as tomadas de decisão no enfrentamento a pandemia da COVID-19 no Ceará mostram-se complexos e amplos, estando em sua maioria alinhados com posicionamentos de paises que obtiveram êxito nesse enfrentamento. Assim, urge a necessidade de estabelecer parcerias efetivas entre atores. serviços e setores instigados, na adoção de recomendações que minimizem os danos da pandemia e viabilizem prontamente o fim do crescimento no número de casos e agravamento destes.

A análise possibilitou reconhecer como fluxo de problemas o processo de interiorização da epidemia no estado e a concentração de caso na capital Fortaleza; como soluções as iniciativas nos setores de saúde, educação, assistência social, economia, trabalho, segurança pública, esporte e cultura; e, por fim, no fluxo de forças políticas, ressalta-se o descontentamento da população e líderes políticos com as atitudes do Presidente e o fortalecimento de parcerias entre setores públicos, privados e esferas de liderança social.

Destarte, o modelo dos múltiplos fluxos de Kingdon demonstrou contribuir na análise de políticas de saúde e das forças empregadas em sua efetivação, apresentadas na forma de fluxos, possibilitando interpretações sobre as problemáticas de saúde e influências das relações políticas, intergovernamentais e da sociedade civil na agenda pública.

Contribuições dos Autores: Concepção do estudo: Machado LDSM, Xavier SPL. Coleta, análise e interpretação dos dados: Machado LDSM, Xavier SPL, Torres FAF, Leite PL, Santos TR, Melo LU, Nunes SF, Silva MRP. Redação e/ou revisão crítica do conteúdo: Machado LDSM, Xavier SPL, Torres FAF, Leite PL, Santos TR, Melo LU, Nunes SF, Silva MRF. Revisão e aprovação final da versão final: Machado LDSM, Xavier SPL, Silva MRP

FINANCIAMENTO: Bolsas de Iniciação Científica e Extensão do Fundo Estadual de Combate à Pobreza (FECOP)- Universidade Regional do Cariri

\section{REFERÊNCIAS}

1.Chaves TSS, Bellei N. SARS-COV-2, o novo Coronavirus: uma reflexão sobre a Saúde Única (One Health) e a importância da medicina de viagem na emergência de novos patógenos [editorial]. Rev Med, São Paulo [Internet]. 2020 [acesso 2020 abr 18]; 99(1):1-4. Disponivel em: http://dx.doi.org/10.11606/issn.1679-9836.v99ilpi-iv

2. Lana RM, Coelho FC, Gomes MFC, Cruz OG, Bastos LS, Villela DAM, Codeço CT. Emergência do novo coronavírus (SARS-CoV-2) e o papel de uma vigilância nacional em saúde oportuna e efetiva. Cad. Saúde Pública [Internet]. 2020 [acesso 2020 abr 18]; 36(3). Disponivel em: DOI: http://dx.doi.org/10.1590/0102-311X00019620
3. Tunãs ITC, Silva ET, Santiago SBS, Maia KD, Silva-Júnior GO. Doença pelo Coronavirus 2019 (COVID-19): Uma abordagem preventiva para Odontologia. Rev. Bras. Odontol [Internet]. 2020 [acesso em 2020 abr 20];77:el766. Disponivel em: http://dx.doi.org/10.18363/ rbo.v77.2020.el766

4. Valero-Cedeño NJ, Mina-Ortiz JB, Veliz-Castro TI, Merchán-Villafuerte KM, Perozo-Mena AJ. COVID-19. La nueva pandemia com muchas lecciones y nuevos retos. Revisión Narrativa. Kasmera [Internet]. 2020 [acesso 2020 abr 22];48(1):e48102042020. Disponivel em: https://dx.doi.org/10.5281/zenodo.3745322

5. Lima DLF, Dias AA, Rabelo RS, Cruz ID, Costa SC, Ni- 
gri FMN, Neri JR. COVID-19 no Estado do Ceará: Comportamentos e crenças na chegada da pandemia. Cien Saude Colet [Internet] (2020/Abr). [acesso em 2020 abr 21]. Disponivel em: http://www.cienciaesaudecoletiva.com.br/artigos/covid19-no-estado-do-ceara-comportamentos-e-crencas-na-chegada-da-pandemia/17540?id=17540

6. Croda JHR, Garcia LP. Resposta imediata da Vigilância em Saúde à epidemia da COVID-19. Epidemiol. Serv. Saúde [Internet]. 2020 [acesso em 2020 abr 18]; 29(1): e2020002.

\section{doi: 10.5123/s1679-49742020000100021.}

7. Ministério da Saúde (BR). Secretaria de Vigilância em Saúde. Boletim Epidemiológico 9 - COE Coronavirus [Internet]. 2020 [acesso em 2020 abr 18]. Disponível em: https://www.irrd.org/covid-19/\#brasil

8. Oliveira JLC, Magalhães AMM, Matsuda LM. Métodos mistos na pesquisa em enfermagem: possibilidades de aplicação à luz de Creswell. Texto Contexto Enferm [Internet]. 2018 [acesso em 2020 abr 10]; 27(2):e0560017. Disponivel em: https://doi.org/10.1590/0104070720180000560017.

9. Kingdon JW. Agendas, alternatives and public policies. Ann Arbor: University of Michigan, 2003.

10. Gottems LBD, Pires MRGM, Calmon PCDP, Alves ED. O modelo dos múltiplos fluxos de Kingdon na análise de políticas de saúde: aplicabilidades, contribuições e limites. Saúde Soc. São Paulo [Internet]. 2013 [acesso em 2020 abr 18]; 22(2):511-520. Disponivel em: https://doi. org/10.1590/S0104-12902013000200020

11. Gibbs G. Análise de dados qualitativos. Porto Alegre: Artmed; 2009. $198 \mathrm{p}$

12. Rafael RMR, Neto M, Carvalho MMB, David HMSL, Acioli S, Faria MGA. Epidemiologia, políticas públicas e pandemia de Covid- 19: o que esperar no Brasil? Rev enferm UERJ [Internet]. 2020 [acesso em 2020 abr 21]; 28. Disponivel em: http://dx.doi.org/10.12957/reuerj.2020.49570

13. Gallasch CH, Cunha ML, Pereira LAS, Silva-Junior JS. Prevenção relacionada à exposição ocupacional do profissional de saúde no cenário de COVID-19. Rev enferm UERJ [Internet]. 2020 [Acesso em 2020 abr 22]; 28. Disponivel em: doi:https://doi.org/10.12957/ reuerj.2020.49596.

14. Burki T. COVID-19 in Latin America. Lancet Infect Dis 2020 [Internet]. 2020 [acesso em 2020 April 23]. Disponivel em: https://doi.org/10.1016/S14733099(20)30303-0

15. Nunes J. A pandemia de COVID-19: securitização, crise neoliberal e a vulnerabilização global. Cad. Saúde Pública [Internet]. 2020 [acesso em 2020 abr 23];36(5), e00063120. Disponivel em: http://dx.doi.org/10.1590/ 0102-311X00063120.

16. Macedo VLM, Vieira LF, Neves RS, Leandro SS. Avaliação da Estratégia Saúde da Família em São Sebastião - Distrito Federal. Enferm. em Foco [Internet]. 2019 [acesso em 2020 abr 23];10(2): 15-21. Disponivel em: http://revista.cofen.gov.br/index.php/enfermagem/article/view/2330/540

17.Leite TRC, Lopes MSV, Maia ER, Cavalcante EGR. Avaliação da estrutura da atenção primária à saúde na atenção à hanseniase. Enferm. Foco [Internet]. 2019 [acesso em 2020 abr 23]; 10 (4): 73-78. Disponivel em: http://revista.cofen.gov.br/index.php/enfermagem/ article/view/2216/608

18.Silva AAM. Sobre a possibilidade de interrupção da epidemia pelo coronavirus (COVID-19) com base nas melhores evidências cientificas disponiveis. Rev Bras Epidemiol [Internet]. 2020 [acesso em 2020 April 23]; 23. Disponivel em: https://dx.doi.org/10.1590/1980549720200021

19. Morosini MVGC, Fonseca AF, Lima LD. Política Nacional de Atenção Básica 2017: retrocessos e riscos para o Sistema Único de Saúde. Saúde Debate [Interrnet]. 2018 [acesso em 2020 abr 21]; 42(116):1124. Disponivel em: https://dx.doi.org/10.1590/01031104201811601

20.Souza ML. The land of the past? Neo-populism, neo-fascism, and the failure of the left in Brazil. Political Geography [Internet]. 2020 [acesso em 2020 abr 23]. Disponivel em: https://dx.doi.org/10.1016/j.polgeo.2020.102186 